\title{
New Solar Composition: The Problem With Solar Models Revisited
}

\author{
Aldo M. Serenelli \\ Max Planck Institute for Astrophysics, Karl Schwarzschild Str. 1, Garching, D-85471, Germany \\ Sarbani Basu \\ Department of Astronomy, Yale University, P.O. Box 208101, New Haven, CT 06520-8101, USA \\ Jason W. Ferguson \\ Department of Physics, Wichita State University, Wichita KS67260-0032, USA \\ and \\ Martin Asplund \\ Max Planck Institute for Astrophysics, Karl Schwarzschild Str. 1, Garching, D-85471, Germany
}

\begin{abstract}
We construct updated solar models with different sets of solar abundances, including the most recent determinations by Asplund et al. (2009). The latter work predicts a larger $(\sim 10 \%)$ solar metallicity compared to previous measurements by the same authors but significantly lower $(\sim 25 \%)$ than the recommended value from a decade ago by Grevesse \& Sauval (1998). We compare the results of our models with determinations of the solar structure inferred through helioseismology measurements. The model that uses the most recent solar abundance determinations predicts the base of the solar convective envelope to be located at $R_{\mathrm{CZ}}=0.724 \mathrm{R}_{\odot}$ and a surface helium mass fraction of $Y_{\text {surf }}=0.231$. These results are in conflict with helioseismology data $\left(R_{\mathrm{CZ}}=0.713 \pm 0.001 \mathrm{R}_{\odot}\right.$ and $\left.Y_{\text {surf }}=0.2485 \pm 0.0035\right)$ at $5-\sigma$ and $11-\sigma$ levels respectively. Using the new solar abundances, we calculate the magnitude by which radiative opacities should be modified in order to restore agreement with helioseismology. We find that a maximum change of $\sim 15 \%$ at the base of the convective zone is required with a smooth decrease towards the core, where the change needed is $\sim 5 \%$. The required change at the base of the convective envelope is about half the value estimated previously. We also present the solar neutrino fluxes predicted by the new models. The most important changes brought about by the new solar abundances are the increase by $\sim 10 \%$ in the predicted ${ }^{13} \mathrm{~N}$ and ${ }^{15} \mathrm{O}$ fluxes that arise mostly due to the increase in the $\mathrm{C}$ and $\mathrm{N}$ abundances in the newly determined solar composition.
\end{abstract}

Subject headings: Sun: helioseismology - Sun: interior - Sun: abundances - neutrinos

\section{Introduction}

Since the solar surface heavy-element content has been revised downwards by Asplund et al. (2005, AGS05), from $(Z / X)_{\odot}=0.0229$ (Grevesse \& Sauva 1998, GS98) to $(Z / X)_{\odot}=0.0165$, the excellent agreement between standard solar model (SSM) predictions and helioseismology determinations of the solar structure (Christensen-Dalsgaard et al. 1996; Bahcall et al. 2001) has been seriously coml. 2004; Bahcall et al. 2005). This discrepancy be- 
tween models and helioseismic inferences has prompted a number of authors to revise the physical inputs of SSMs (Montalbán et al. 2004; Basu \& Antia 2004; Bahcall et al. 2005; Guzik 2006; Christensen-Dalsgaard et al. 2009), and to question the revision of the solar abundances, particularly those of $\mathrm{C}, \mathrm{N}, \mathrm{O}, \mathrm{Ne}$ and Ar whose fractional abundances cannot be determined from meteoritic samples Antia \& Basu 2005, 2006; Bahcall et al. 2005; Delahaye \& Pinsonneault 2006; Basu \& Antia 2008).

Very recently, Asplund et al. 2009, hereafter AGSS09) have done a complete revision of the solar photospheric abundances for nearly all elements, including a new 3D hydrodynamical solar atmosphere model with improved radiative transfer and opacities (Trampedach et al. 2009). The predictions from this 3D model have been shown to agree remarkably well with various observational constraints, including the atmospheric thermal structure as judged from continuum centerto-limb variation and detailed line profile shapes (Pereira et al. 2009a,b). The newly determined solar abundances lead to $(Z / X) \odot=0.0178$, higher than the AGS05 value but still well below older determinations, e.g. GS98.

In this Letter we present a series of new SSM calculations using solar compositions from GS98, AGS05, and the newly determined solar abundances by AGSS09. All the models incorporate new refinements in the input physics, so that models presented here with the older compositions (GS98 and AGS05) represent updated versions of previous solar model calculations Bahcall et al. 2005). For each model, we compare our results with helioseismological determinations of solar properties and also give the predicted solar neutrino fluxes. Additionally, and motivated by the new AGSS09 composition, we determine the factor by which radiative opacities in the solar interior should be increased to solve the solar abundance problem following the scheme presented by Christensen-Dalsgaard et al. (2009).

\section{Calculations and Results}

Solar models in this work have been computed with a modified version of GARSTEC (Weiss \& Schlatt 2008) that uses the nuclear en- ergy generation routine exportenergy.1. Element diffusion in the solar interior is included according to Thoul et al. (1994). Radiative opacities are from the Opacity Project, complemented at low temperatures with those from Ferguson et al. (2005). Specific sets of opacities have been computed for each of the solar compositions used in this paper (see below). With respect to previous works, e.g. Bahcall et al. (2005, 2006), the changes in the input physics are: a revised version of the OPAL equation of state 2 (EOS) that corrects errors in the 2002 OPAL EOS tables from Rogers \& Nayfonov (2002) (our previous choice), and updated values of two important nuclear astrophysical factors, $\mathrm{S}_{34}$ (Costantini et al. 2008) and $\mathrm{S}_{1,14}$ (Marta et al. 2008), the latest determinations by the LUNA experiment.

We have computed solar models using three different basic solar abundances. Two models employ previous solar abundance compilations (GS98 and AGS05) and show small differences with respect to models with the same abundances presented elsewhere, e.g. Bahcall et al. (2005). The changes originate from the use of the updated EOS and cross-sections for nuclear reactions mentioned above. A third SSM has been computed adopting the new solar composition determined by AGSS09. The most important results in this work are related to this model. The choice of the abundance scale (meteoritic or photospheric) deserves a short discussion. While AGSS09 find the average difference between photospheric and meteoritic abundances to be $0.00 \pm 0.04$ dex, a few elements relevant to detailed solar modeling show comparable or slightly larger deviations. This is the case for $\mathrm{Mg}, \mathrm{Ca}$, and Fe for which differences between the two scales are $0.07,0.05$, and 0.05 dex respectively, photospheric values being larger. Given the historical robustness and higher accuracy of meteoritic determinations of abundance ratios, and the present excellent overall agreement with photospheric abundances, we adopt for AGSS09 me-

\footnotetext{
${ }^{1}$ Publicly available at http://www.sns.ias.edu/ jnb

${ }^{2}$ http://adg.llnl.gov/Research/OPAL/EOS_2005/. OPAL EOS uses relative metal abundances for $\mathrm{C}, \mathrm{N}, \mathrm{O}$, and $\mathrm{Ne}$ close to Grevesse \& Noels (1993) and abundances of heavier elements are added to Ne. Differences in relative abundances of metals with more recent solar abundance compilations have negligible influence in the global properties of solar models, provided the correct overall metallicity is used (Gong et al. 2001; Rogers \& Navfonov 2002).
} 
teoritic abundances for refractory elements as the standard choice for our solar models. We also investigate, however, the use of adopting the photospheric values throughout. This is also consistent with the adoption of meteoritic scales in previous works on solar models (e.g. Bahcall et al. 2005, 2006). The abundances for the different solar compositions used in this paper are given in Table 1 . in particular for those elements entering the calculation of radiative opacities.

Models have been evolved from the pre-main sequence to the present-day solar system age, $\tau_{\odot}=$ $4.57 \mathrm{Gyn} 3$. The main characteristics of the models are listed in Table 2. The second and third columns give the present-day heavy elements to hydrogen mass ratio and the surface metallicity. From the fourth to the seventh columns we present quantities directly related to helioseismology: surface helium mass fraction $Y_{\text {surf }}$, depth of the convective zone $R_{\mathrm{CZ}} / \mathrm{R}_{\odot}$, and the average rms difference between model and solar sound speed and density profiles, $\langle\delta c / c\rangle$ and $\langle\delta \rho / \rho\rangle$ respectively. Columns eighth and ninth give the central helium mass fraction $Y_{\mathrm{c}}$ and metallicity $Z_{\mathrm{c}}$ at $\tau_{\odot}$. Finally, the last three columns give the initial composition of the models and the mixing length parameter.

Results for the GS98 and AGS05 models are very similar to those presented in Bahcall et al. (2005); the improved EOS leads to changes in the sound speed and density profiles about one order of magnitude smaller than the differences between solar models and helioseismic inferences, while changes in nuclear cross sections only affect predictions for neutrino fluxes that are discussed in detail below. Helioseismically derived values for $R_{\mathrm{CZ}} / \mathrm{R}_{\odot}$ and $Y_{\text {surf }}$ and are $0.713 \pm 0.001$ (Basu \& Antia 1997) and $0.2485 \pm 0.0035$ (Basu \& Antia 2004) respectively. The GS98 model predicts $R_{\mathrm{CZ}} / R_{\odot}$ with the right value compared to helioseismology, while the AGS05 model shows a $15-\sigma$ discrepancy. For $Y_{\text {surf }}$ the situation is analogous: GS98 value differs from the helioseismology determination by 1.8- $\sigma$ while for AGS05 the discrepancy is 5.5- $\sigma$.

For the SSM that adopts the newly determined solar composition AGSS09, $R_{\mathrm{CZ}} / \mathrm{R}_{\odot}$ and

\footnotetext{
${ }^{3}$ Detailed structure of solar models at $\tau \odot$ presented in this work can be found in http://www.mpa-garching.mpg.de/ aldos
}

$Y_{\text {surf }}$ show some improvements with respect to the AGS05 model, but still far away from the helioseismology values by $11-\sigma$ and $5-\sigma$ respectively. The slight increase in oxygen abundance ( $0.03 \mathrm{dex})$ and the larger change in neon (0.09 dex) contribute to enhance the opacity below the convective zone (CZ), deepening its inner boundary and decreasing the mismatch with the solar sound speed at the same time. This is illustrated in the top panel of Figure 1 where the relative difference in sound speed is shown for the models considered in this work. The peak in the profile of the sound speed difference, right below the $\mathrm{CZ}$, is $\sim 1 \%$ for the AGSS09 composition, an improvement with respect to AGS05 but still significantly higher than that for the GS98 model. The overall agreement with the solar sound speed, as derived by inversions and indicated by $\langle\delta c / c\rangle$, is a factor of four times worse for the AGSS09 model than for GS98. For the latter model, $\langle\delta c / c\rangle$ is almost unchanged compared to results from previous works. However, a detailed comparison of the sound speed profiles shown in Figure 1 with those presented in Bahcall et al. (2005) unveils some differences, more evident below $R \approx 0.6 \mathrm{R}_{\odot}$. These changes are not related to the improved physics adopted in the new models, but rather the result of using better data for low-degree $(\ell \leq 3)$ modes that penetrate the solar core (see Basu et al. 2009 for details). In the same figure, results for density inversions are shown in the bottom panel. Again, the AGSS09 composition leads to an improvement in the agreement with helioseismology compared to the AGS05 model, but still far from the results obtained for the GS98 composition.

As already mentioned, meteoritic and photospheric abundances in AGSS09 agree with each other very well, but a few elements show differences that could have potential impact on details in the solar structure. To quantify this assertion, we have computed an additional SSM using only the photospheric abundances given in AGSS09, for which $(Z / X)_{\odot}=0.0181$. The main characteristics of this model, identified as AGSS09ph, are given in the last entry of Table 2. Compared to the model with the meteoritic abundances, AGSS09ph performs somewhat better in terms of helioseismological quantities as inferred from the results summarized in the table, with discrepancies with the measured depth of the $\mathrm{CZ}$ and surface helium 
abundance of the order of 9- $\sigma$ and 4- $\sigma$ respectively. The sound speed and density profiles are shown as dotted lines in Figure 1. The changes with respect to our standard AGSS09 (meteoritic scale) model changes are mostly due to the larger $\mathrm{Mg}$ and $\mathrm{Fe}$ photospheric abundances (0.07 and 0.05 dex, respectively) that enhance the opacity in the radiative interior; the fractional increase in opacity is larger than the fractional increase in the overall metallicity (note the largest improvement in the sound speed, for instance, occurs at $R \approx 0.5 \mathrm{R}_{\odot}$, the region where the contribution of $\mathrm{Mg}$ to the opacity is largest). Our results show that adoption of the photospheric scale gives slight improvements in the solar model predictions. However, since the uncertainties in the meteoritic abundances typically are smaller than the corresponding ones for the photospheric determinations the meteoritic scale is our preferred choice for solar abundances (with the exception of the volatile elements that are depleted in meteorites). This is reinforced by the historical robustness of meteoritic abundance determinations.

Low-degree helioseismology $(\ell \leq 3)$ can be used to derive seismic information on the solar core, particularly by using the so-called separation ratios as described by Roxburgh \& Vorontsov (2003). Chaplin et al. (2007) have used the separation ratios constructed with very precise frequencies of low- $\ell$ modes measured by the Birmingham Solar-Oscillations Network (BiSON) to constrain properties of the solar core. They showed the discrepancy in solar models constructed with AGS05 composition extends all the way to the solar core and it is not just related to deficiencies in the modeling of the solar outer layers, in the convective envelope. Here we compare the observed separation ratios to those computed for our solar models; results are displayed in Figure 2. As with other helioseismology indicators, the GS98 model performs much better than the AGS05 model. The adoption of the AGSS09 composition in the SSM has very small influence in the core structure of the model, as it practically overlaps the AGS05 model. Results for the AGSS09ph model closely resemble those from AGSS09 and, for clarity, are not shown in Figure 2. As discussed in Basu et al. (2007), values of the separation ratios are closely related to the quantity $1 / r(d c / d r)$ integrated over the solar structure. Differences in this quantity between models with AGS05 and AGSS09 (both meteoritic and photospheric) compositions are very small and only present very close to the center $\left(r<0.05 R_{\odot}\right)$ and thus have a very small impact on the separation ratios.

Solar neutrino fluxes for the models are listed in Table 3. The new astrophysical factor $S_{34}$ (Costantini et al. 2008) is 7\% larger than previous determination and it is responsible for the larger ${ }^{7} \mathrm{Be}$ and ${ }^{8} \mathrm{~B}$ fluxes of models GS98 and AGS05 with respect to those published in Bahcall et al. (2006) with the same solar compositions. Similarly, the somewhat smaller $(7.6 \%) S_{1,14}$ value recently published by the LUNA collaboration (Marta et al. 2008) leads to a proportional reduction in the ${ }^{13} \mathrm{~N}$ and ${ }^{15} \mathrm{O}$ fluxes. The increase in metallicity in the AGSS09 solar composition, compared to AGS05, is only partially reflected in the changes of the neutrino fluxes. This is so because elements that more strongly affect the solar core temperature ( $\mathrm{Si}, \mathrm{S}$, and $\mathrm{Fe}$ ) have the same abundance in AGS05 and the meteoritic AGSS09 scale. The increase by $0.09 \mathrm{dex}$ in Ne and by $0.22 \mathrm{dex}$ in Ar are the most important changes in abundances influencing the core temperature. This is reflected, for instance, in the $\sim 4 \%$ increase in the ${ }^{8} \mathrm{~B}$ flux from AGS05 to AGSS09. ${ }^{13} \mathrm{~N}$ and ${ }^{15} \mathrm{O}$ fluxes, that depend almost linearly in the added abundance of carbon and nitrogen show larger changes (of the order of $\sim 12-14 \%$ ) due to the 0.03 and 0.05 dex larger abundances of these elements in the new AGSS09 abundances. Differences in the neutrino fluxes between the AGSS09 and the GS98 models are of the order of $10 \%$ for ${ }^{7} \mathrm{Be}, 20 \%$ for ${ }^{8} \mathrm{~B}$ and $38 \%$ for the added ${ }^{13} \mathrm{~N}$ and ${ }^{15} \mathrm{O}$ fluxes. For the sake of completeness we present the neutrino fluxes for the AGSS09ph model. The effect of the increased iron abundance is readily noticeable particularly in the larger ${ }^{8} \mathrm{~B}$ flux compared to AGSS09 model and, to a lesser extent in the $\mathrm{CNO}$ and ${ }^{7} \mathrm{Be}$ fluxes. The possibilities that current and future neutrino experiments offer to constrain the solar core metallicity are beyond the scope of this paper and are discussed elsewhere (Haxton \& Serenelli 2008; Peña-Garay \& Serenelli 2008).

Qualitatively, the AGSS09 abundances do not change the picture that emerged with the previous set of solar abundances, AGS05: SSMs constructed with abundances derived from the most sophisticated solar atmosphere models and up-to- 
date atomic data conflict with all helioseismology inferences of solar structure. Potential solutions to the solar abundance problem analyzed by different authors, none of them successful in restoring the agreement between SSM and helioseismology measurements, still face the same problems if the AGSS09 composition is used instead of AGS05. In this regard, it is particularly informative the work by Delahaye \& Pinsonneault (2006), which show in the $R_{\mathrm{CZ}}-Y_{\text {surf }}$ plane the direction in which model predictions change when modifications in their input physics are applied. It is not a simple task to find deficits in the models that produce simultaneously changes in both $R_{\mathrm{CZ}}$ and $Y_{\text {surf }}$ in the right direction, except for restoring the metallicity to a larger value, comparable to that of GS98. Quantitatively, however, the disagreement is less severe with AGSS09 composition and, motivated by this fact, it is worth taking another look at the opacity deficit induced by the AGSS09 abundances. We have done a similar analysis to the one presented by Christensen-Dalsgaard et al. (2009) to check by how much opacities in the AGSS09 model have to be increased to recover the level of agreement with helioseismology that the GS98 model gives. We find that in the central regions the required change is $\sim 5 \%$ ( $2 \%$ with AGSS09ph), very close to what Christensen-Dalsgaard et al. (2009) found. The magnitude of the change increases smoothly outwards and reaches $\sim 15 \%$ (12\% with AGSS09ph) at the bottom of the CZ. This requirement is smaller by almost a factor of two than that found by Christensen-Dalsgaard et al. (2009), very likely because they used the S-model (which used the higher $(Z / X)_{\odot}=0.0245$ from Grevesse \& Noels 1993) as their reference model and one with the AGS05 solar composition, while we have used GS98 and AGSS09 (see Serenelli 2010 for a more thorough discussion). We note the required changes are much larger than differences found between OP and OPAL opacities in the radiative solar interior. It would be interesting, as pointed out by Christensen-Dalsgaard et al. (2009), to find other observable implications that changes of $12-15 \%$ in the radiative opacity at temperatures of a couple to a few millon degrees would have, independently of those from solar models.

\section{Summary}

We have computed new standard solar models that incorporate the most up-to-date input physics, including the updated OPAL equation of state and the most recent determinations of the astrophysical factors for the important ${ }^{3} \mathrm{He}\left({ }^{4} \mathrm{He}, \gamma\right){ }^{7} \mathrm{Be}$ and ${ }^{14} \mathrm{~N}(\mathrm{p}, \gamma){ }^{15} \mathrm{O}$ reactions. We have used three different sets of solar abundances: GS98, AGS05 and the newly determined AGSS09. We have found that the updated physical inputs have very little effect on the properties of solar models, both in terms of solar structure and in the neutrino fluxes. The most important results in this work are those from the model that adopts the new set of solar abundances, AGSS09. The new abundances are determined by using an improved solar atmosphere model and atomic data, and a more careful selection of spectroscopic lines. The resulting solar metallicity is somewhat larger than that from AGS05. This is reflected in the helioseismological properties of the solar model. For the AGSS09 model, the sound speed and density profiles, the predicted surface helium abundance and the depth of the convective zone are still in conflict with helioseismology data, although the disagreement is less severe than for the AGS05 model. Still, results are far from the excellent match found with the GS98 composition. Finally, we have found that if radiative opacities were to be modified to restore the agreement between solar models (with AGSS09 composition) and helioseismology, the required changes are $\approx 12-15 \%$ right below the convective zone with a smooth decrease towards the central regions, where changes should be $2-5 \%$.

AMS thanks Maria Bergemann for insightful discussions on the physics of non-LTE effects in the formation of spectral lines. SB is partially supported by NSF grant ATM-0348837. We thank the anonymous referee for useful suggestions and comments.

\section{REFERENCES}

Ahmad, Q. R., et al. 2002, Physical Review Letters, 89, 011301

Aharmim, B., et al. 2005, Phys. Rev. C, 72, 055502 
Aharmim, B., et al. 2008, Physical Review Letters, 101,111301

Antia, H. M., \& Basu, S. 2005, ApJ, 620, L129

Antia, H. M., \& Basu, S. 2006, ApJ, 644, 1292

Arpesella, C., et al. 2008, Physical Review Letters, 101, 091302

Asplund, M., Grevesse, N., \& Sauval, J. 2005, Cosmic Abundances as Records of Stellar Evolution and Nucleosynthesis, 336, 25

Asplund, M., Grevesse, N., Sauval, J., \& Scott, P. 2009, ARA\&A, 47, 481

Badnell, N. R., Bautista, M. A., Butler, K., Delahaye, F., Mendoza, C., Palmeri, P., Zeippen, C. J., \& Seaton, M. J. 2005, MNRAS, 360, 458

Bahcall, J. N., Basu, S., Pinsonneault, M., \& Serenelli, A. M. 2005, ApJ, 618, 1049

Bahcall, J. N., Basu, S., \& Serenelli, A. M. 2005, ApJ, 631, 1281

Bahcall, J. N., Pinsonneault, M., \& Basu, S. 2001, ApJ, 555, 990

Bahcall, J. N., Serenelli, A. M., \& Basu, S. 2006, ApJS, 165, 400

Bahcall, J. N., Serenelli, A. M., \& Basu, S. 2005, ApJ, 621, L85

Bahcall, J. N., Serenelli, A. M., \& Pinsonneault, M. 2004, ApJ, 614, 464

Basu, S., \& Antia, H. M. 1997, MNRAS, 287, 189

Basu, S., \& Antia, H. M. 2004, ApJ, 606, L85

Basu, S., \& Antia, H. M. 2008, Phys. Rep., 457, 217

Basu, S., Chaplin, W. J., Elsworth, Y., New, R., Serenelli, A. M., \& Verner, G. A. 2007, ApJ, 655,660

Basu, S., Chaplin, W. J., Elsworth, Y., New, R., \& Serenelli, A. M. 2009, ApJ, 699, 1403

Chaplin, W. J., Serenelli, A. M., Basu, S., Elsworth, Y., New, R., \& Verner, G. A. 2007, ApJ, 670, 872
Christensen-Dalsgaard, J. C., et al. 1996, Science, 272,1286

Christensen-Dalsgaard, J., di Mauro, M. P., Houdek, G., \& Pijpers, F. 2009, A\&A, 494, 205

Costantini, H., et al. 2008, Nuclear Physics A, 814, 144

Delahaye, F., \& Pinsonneault, M. H. 2006, ApJ, 649,529

Ferguson, J. W., Alexander, D. R., Allard, F., Barman, T., Bodnarik, J. G., Hauschildt, P. H., Heffner-Wong, A., \& Tamanai, A. 2005, ApJ, 623,585

Gong, Z., Däppen, W., \& Nayfonov, A. 2001, ApJ, 563,419

Grevesse, N., \& Noels, A. 1993, Origin and Evolution of the Elements, 15

Grevesse, N., \& Sauval, A. J. 1998, Space Science Reviews, 85, 161

Guzik, J. A. 2006, Proceedings of SOHO 18/GONG 2006/HELAS I, Beyond the spherical Sun, 624, 17

Haxton, W. C., \& Serenelli, A. M. 2008, ApJ, 687, 678

Marta, M., et al. 2008, Phys. Rev. C, 78, 022802

Montalbán, J., Miglio, A., Noels, A., Grevesse, N., \& di Mauro, M. P. 2004, in ESA Special Publication, Vol. 559, SOHO 14 Helio- and Asteroseismology: Towards a Golden Future, ed. D. Danesy, 574-+

Peña-Garay, C., \& Serenelli, A. 2008, arXiv:0811.2424

Pereira, T. M. D., Asplund, M., Trampedach, R., \& Collet, R. 2009, A\&A, submitted

Pereira, T. M. D., Kiselman, D., \& Asplund, M. 2009, A\&A, submitted

Rogers, F. J., \& Nayfonov, A. 2002, ApJ, 576, 1064

Roxburgh, I. W., \& Vorontsov, S. V. 2003, A\&A, 411,215 
Serenelli, A. 2010, Ap\&SS in Synergies Between Solar and Stellar Modelling, ed. M. Marconi, D. Cardini \& M. P. Di Mauro, in preparation

Thoul, A. A., Bahcall, J. N., \& Loeb, A. 1994, ApJ, 421, 828

Trampedach, R., Asplund, M., Hayek, W., \& Collet, R. 2009, A\&A, to be submitted

Weiss, A., \& Schlattl, H. 2008, Ap\&SS, 316, 99

This 2-column preprint was prepared with the AAS IATEX macros v5.2. 
TABLE 1

Adopted SOLAR CHEMICAL COMPOSITIONS.

\begin{tabular}{lcccc}
\hline \hline & \multicolumn{3}{c}{$\log \epsilon$} \\
\cline { 2 - 5 } Element & GS98 & AGS05 $^{a}$ & AGSS09 $^{a}$ & AGSS09ph $^{b}$ \\
\hline $\mathrm{C}$ & 8.52 & 8.39 & 8.43 & 8.43 \\
$\mathrm{~N}$ & 7.92 & 7.78 & 7.83 & 7.83 \\
$\mathrm{O}$ & 8.83 & 8.66 & 8.69 & 8.69 \\
$\mathrm{Ne}$ & 8.08 & 7.84 & 7.93 & 7.93 \\
$\mathrm{Na}$ & 6.32 & 6.27 & 6.27 & 6.24 \\
$\mathrm{Mg}$ & 7.58 & 7.53 & 7.53 & 7.60 \\
$\mathrm{Al}$ & 6.49 & 6.43 & 6.43 & 6.45 \\
$\mathrm{Si}$ & 7.56 & 7.51 & 7.51 & 7.51 \\
$\mathrm{~S}$ & 7.20 & 7.16 & 7.15 & 7.12 \\
$\mathrm{Ar}$ & 6.40 & 6.18 & 6.40 & 6.40 \\
$\mathrm{Ca}$ & 6.35 & 6.29 & 6.29 & 6.34 \\
$\mathrm{Cr}$ & 5.69 & 5.63 & 5.64 & 5.64 \\
$\mathrm{Mn}$ & 5.53 & 5.47 & 5.48 & 5.43 \\
$\mathrm{Fe}$ & 7.50 & 7.45 & 7.45 & 7.50 \\
$\mathrm{Ni}$ & 6.25 & 6.19 & 6.20 & 6.22 \\
\hline
\end{tabular}

Note.-Abudances given as $\log \epsilon_{i} \equiv \log N_{i} / N_{H}+12$.

${ }^{a}$ The adopted abundances are the recommended solar photospheric abundances for the volatile elements $(\mathrm{C}, \mathrm{N}, \mathrm{O}, \mathrm{Ne}$ and $\mathrm{Ar}$ ) and the CI chondritic meteoritic values for the remaining elements. ${ }^{b}$ The adopted abundances are the recommended solar photospheric abundances throughout. 
TABLE 2

MAIN CHARACTERISTICS OF SOLAR MODELS.

\begin{tabular}{lccccccccccc}
\hline \hline Model & $(Z / X)_{\text {surf }}$ & $Z_{\text {surf }}$ & $Y_{\text {surf }}$ & $R_{\mathrm{CZ}} / R_{\odot}$ & $\langle\delta c / c\rangle$ & $\langle\delta \rho / \rho\rangle$ & $Y_{\mathrm{c}}$ & $Z_{\mathrm{c}}$ & $Y_{\text {ini }}$ & $Z_{\text {ini }}$ & $\alpha_{\mathrm{MLT}}$ \\
\hline GS98 & 0.0229 & 0.0170 & 0.2423 & 0.713 & 0.0010 & 0.011 & 0.6330 & 0.0201 & 0.2721 & 0.0187 & 2.15 \\
AGS05 & 0.0165 & 0.0126 & 0.2292 & 0.728 & 0.0049 & 0.048 & 0.6195 & 0.0149 & 0.2593 & 0.0139 & 2.10 \\
AGSS09 & 0.0178 & 0.0134 & 0.2314 & 0.724 & 0.0038 & 0.040 & 0.6220 & 0.0160 & 0.2617 & 0.0149 & 2.09 \\
AGSS09ph & 0.0181 & 0.0136 & 0.2349 & 0.722 & 0.0031 & 0.033 & 0.6263 & 0.0161 & 0.2653 & 0.0151 & 2.12 \\
\hline
\end{tabular}


TABLE 3

PRedicted NEUtrino FLuXes.

\begin{tabular}{lcccccccc}
\hline \hline Model & pp & pep & hep & ${ }^{7} \mathrm{Be}$ & ${ }^{8} \mathrm{~B}$ & ${ }^{13} \mathrm{~N}$ & ${ }^{15} \mathrm{O}$ & ${ }^{17} \mathrm{~F}$ \\
\hline GS98 & 5.97 & 1.41 & 7.91 & 5.08 & 5.88 & 2.82 & 2.09 & 5.65 \\
AGS05 & 6.04 & 1.44 & 8.24 & 4.54 & 4.66 & 1.85 & 1.29 & 3.14 \\
AGSS09 & 6.03 & 1.44 & 8.18 & 4.64 & 4.85 & 2.07 & 1.47 & 3.48 \\
AGSS09ph & 6.01 & 1.43 & 8.10 & 4.79 & 5.22 & 2.15 & 1.55 & 3.70 \\
\hline
\end{tabular}

Note.-Neutrino fluxes are given in units of $10^{10}(\mathrm{pp}), 10^{9}\left({ }^{7} \mathrm{Be}\right)$, $10^{8}$ (pep, $\left.{ }^{13} \mathrm{~N},{ }^{15} \mathrm{O}\right), 10^{6}\left({ }^{8} \mathrm{~B},{ }^{17} \mathrm{~F}\right)$ and $10^{3}$ (hep) $\mathrm{cm}^{-2} \mathrm{~s}^{-1}$. Direct measurement by the Borexino experiment of the ${ }^{7} \mathrm{Be}$ flux gives $5.18 \pm 0.51 \times 10^{9} \mathrm{~cm}^{-2} \mathrm{~s}^{-1}$ after 192 days of data Arpesella et al. 2008). For ${ }^{8} \mathrm{~B}$, and until the SNO collaboration presents a combined analysis of the three phases of the experiment, a simple weighed average of the three phases of the SNO experiment Ahmad et al. 2002; Aharmim et al. 2005, 2008) gives $5.18 \pm 0.29 \times 10^{6} \mathrm{~cm}^{-2} \mathrm{~s}^{-1}$ for this flux. 

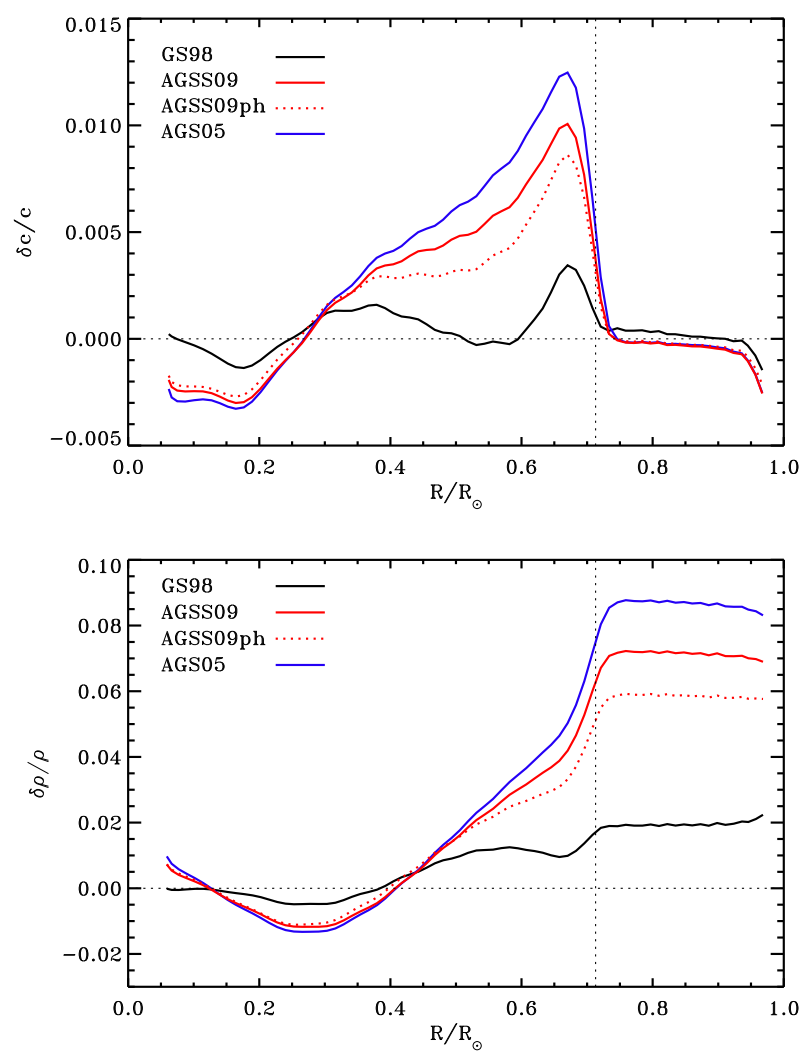

Fig. 1.- Relative sound speed $\delta c / c$ and density $\delta \rho / \rho$ differences, in the sense (Sun Model)/Model, between solar models and helioseismological results. Details on the inversion procedure and data used, as well as the reference sound speeds and densities are given in Basu et al. (2009). 

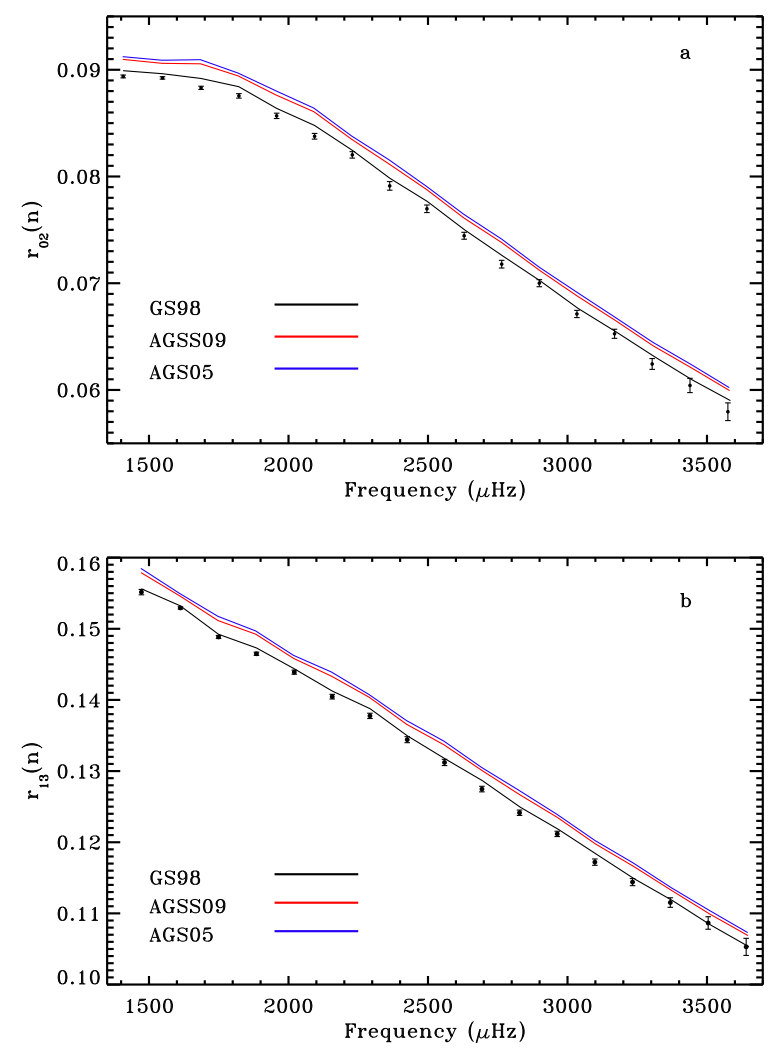

Fig. 2.- Separation ratios. Comparison between values determined from BiSON data and the solar models presented in this work. 